\title{
Modeling and Implementation of AC Electrical Capacitance Tomography
}

\author{
K. Manikandan, S. Sathiyamoorthy \\ Department of Electronics and Instrumentation Engineering J.J. College of Engineering and Technology, \\ Anna University, Chennai, India \\ Email: kmanirec@gmail.com
}

How to cite this paper: Manikandan, $\mathrm{K}$. and Sathiyamoorthy, S. (2016) Modeling and Implementation of AC Electrical Capacitance Tomography. Circuits and Systems, 7, 3818-3830.

http://dx.doi.org/10.4236/cs.2016.711319

Received: April 9, 2016

Accepted: May 5, 2016

Published: September 19, 2016

Copyright $\odot 2016$ by authors and Scientific Research Publishing Inc. This work is licensed under the Creative Commons Attribution International License (CC BY 4.0).

http://creativecommons.org/licenses/by/4.0/

(c) (i) Open Access

\begin{abstract}
Electrical Capacitance Tomography (ECT) determines the dielectric permittivity of the interior object depending on the measurements of exterior capacitance. Generally, the electrodes are placed outside the PVC cylinder where the medium to be imaged is present; but in ECT using inter-electrode capacitance measurements can be achieved by placing inside of the dielectric medium. In the proposed ECT system, the ECT sensor is modeled using ANSYS software and the model is implemented in real ECT system. For each step of measurement, a stable AC signal is applied to a pair of electrodes that form a capacitor. The novel system is to measure the capacitance range variation in picofarad and the corresponding voltage ranges from 1 volt to 4 volts. The switching speed of all combinational electrodes is implemented using embedded system to achieve higher speed performance of AC ECT system which eliminates the drift and stray capacitance error. This is yielding the original image of unknown multiphase medium inside the electrodes using Lab VIEW. This paper investigates several advantages such as improved overall system performance; simple structure, avoids stray capacitance effect, reduces the drift problem and achieves high signal to noise ratio.
\end{abstract}

\section{Keywords}

Electrical Capacitance Tomography, ANSYS, Lab VIEW, Embedded System, AC Signal

\section{Introduction}

ECT is a process of recovery of the image depending on the capacitance change technique. An ECT measures the capacitance from multiple electrodes which surround a dielectric process. There are a various number of capacitive electrodes mounted around 
the measured object, to find the distribution of dielectric capacitance between the electrodes measured. Capacitance sensor is a very promising device for the flow process parameters measurement. Due to the variation in the percentage of air and liquid flowing in the pipe, the mean dielectric constant changes in between the electrode. This results change in the capacitance of the sensor. The two-phase flow measurement uses 8-electrode ECT system [1]-[4], a new sensing system, to calculate the capacitance range in FEM to farads/micro volt range. The electrical connections are made through small holes drilled in the pipe wall. The performance of an ECT sensor was analyzed numerically for the range of dielectric material $\left(1 \leq \varepsilon_{r} \leq 80\right)$ and geometrical parameters. Measurements performed by the ECT system are based on a two-point calibration procedure [5] [6]; the source signal is simultaneously given to all electrodes but the detecting circuit is controlled externally. The potential on the electrode is digitally controlled and the excitation field of the sensor is totally flexible. Applying multiple excitation signals to electrodes can improve overall ECT system performance [7]-[9]; all the electronic circuitry is included on a custom silicon integrated circuit that is implemented using a high voltage CMOS technology. This chip includes charge/discharge circuit; programmable gain offset compensation, ADC and DAC circuit and interfacing unit and also improves the speed of inter-electrodes capacitance by applying multiple excitation signals [10], the CMOS circuit design of capacitance sensor for detecting changes of capacitance due to the incidence of particles or bubbles on the electrodes, determining the capacitance from the permittivity patterns and describes linear back projections methods of image reconstruction [11]-[14]. In this we discuss, sensitive field models of typical flow were established and simulated, using ANSYS which is finite analysis software. The gained capacitance values from ANSYS would be used to be as the sample data. For real time, the ECT is a method for the determination of the dielectric permittivity in the interior of the object based on exterior capacitance measurements.

The proposed system is shown in Figure 1. An AC ECT system consists of five basic parts: 1) sensor system, 2) excitation source generation, 3) embedded system, 4) signal processing parts, 5) interface and monitoring. The sensor consists of a set of electrodes symmetrically fixed outdoor, on the insulation pipe. The sensing electrodes measure the capacitance for every probable electrode combination. The system is made up of

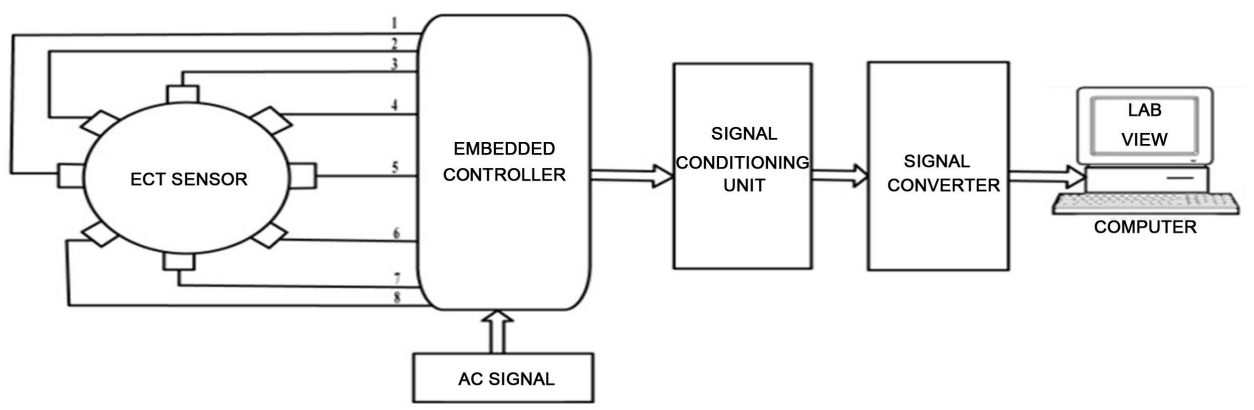

Figure 1. Block diagram of ECT system. 
array of electrodes and embedded controllers, which help increase the system's speed. The embedded system is used to interface the excitation source with the combination electrodes. In a dynamic model like the flow of water or any type of liquids, etc., while entering into the system, one among the eight electrodes is used as the source which is going to sense the flow of liquids and the remaining electrodes act as the detector. The detected signal is in the form of current. The output obtained from the sensor can be converted into a voltage with the help of a current to signal conditioning circuit. The signal is implemented in the NI DAQ. This is used to convert a digital form from the sensor to an analog signal. Finally, the output signal is obtained from a PC with LAB VIEW. An AC stable model is a method of sine wave excitation. The source signal enters the electrodes with the help of an AT mega controller to increase the switching speed. The embedded system activates the analog switches to improve the overall speed of the system.

\section{Design and Analysis of ECT Sensor by ANSYS}

\subsection{Principle of a Capacitance Sensor}

The principle of this sensor is that, when the dielectric medium changes, the capacitance value also gets changed. Basically any two closest conductors can be considered as a capacitor, and dissimilar dielectric properties between the conductors will form various capacitor values. The sensors normally consist of two electrode plates and the capacitance. The capacitance and permittivity distribution relationship is governed by the subsequent equation.

$$
C=\frac{Q}{V}=\varepsilon_{0} \varepsilon_{r} \frac{A}{D}
$$

where,

$\varepsilon_{0}$ - The permittivity of vacuum,

$\varepsilon_{r}$ - The relative permittivity of the material inside the sensor,

$A$-The area of the plates,

$D$-The distance between the two plates.

$\mathcal{E}_{0}$ and $\mathcal{E}_{r}$ are the global standard of the fluid dielectric property over the whole sensing volume of the sensor or better known as permittivity. If the distance of the plates and them areas are known and by measuring the capacitance; we can effectively measure the dielectric constant. In this case, the permittivity in between the electrodes is directly proportional to capacitance value.

\subsection{ANSYS Modelling}

The Capacitor senor is designed using the ANSYS software. This sensor is mainly constructed for imaging multiphase flow such as water-air, oil-air, oil-gas, and gas-water mixtures. Thus, we can maintain an optimum ratio between the mixtures flowing inside the pipe. The finite element technique is utilized to determine the sensitivity distribution across all pixels up to the boundary of the pipe and this is done in the ANSYS also called System Analysis Software. Capacitance sensor is a soft field sensor. In addition, 
the number of possible independent measurements is very small compared to the number of pixels required for an acceptable image. These problems make it difficult to reconstruct good quality images. It is based on first obtaining the sensitivity distributions (or sensitivity map) for all electrode pairs, and then linearly super imposing the normalized capacitances using the sensitivity maps as the weighting factors to obtain images. The sensor is designed by using System Analysis Software. In a typical system, 8 electrodes are symmetrically mounted outside a cylindrical insulating pipe. As we acquainted with, the capacitance varied with following parameter as they are dielectric permittivity, the distance between the plates and, the area of the plates. By this technique, the area and the distance between the electrodes are reserved constant, so the dielectric permittivity of the medium indoor the pipe is the only parameter that manipulate the capacitance.

According to given parameters the capacitive sensor is designed in the ANSYS shown in Figure 2.

Pipe outer diameter $=71.5 \mathrm{~mm}$

Pipe inner diameter $=70 \mathrm{~mm}$

Total length of the electrode $=300 \mathrm{~mm}$

Width of the single electrode $=1 \mathrm{~mm}$

During the stage of a scanning frame, an input AC excitation signal is give to one of the source electrodes and the rest of 7 electrodes act as detector in the 8 electrodes ECT system. Consequently, the voltage potential at every one of the detector electrodes is deliberate, one at a time to determine the inter-electrode capacitance. Variation in material distribution is determined from the variation in there capacitance value measured. Permittivity distribution image that represents the distribution of materials can be get back from the capacitance. In this paper, an eight-electrode arrangement is taken as an example, and capacitance measurements are the following steps. A voltage signal is primary applied to electrode 1 and the entire staying electrodes are at ground potential. Now the capacitance is calculated across each couple of electrodes. Electrode 1 is taken as one in the pair in all combinations that is (1-1), (1-2), (1-3)... so on. Next electrode 2 is energized and all the balancing electrodes are kept at earth potential, and

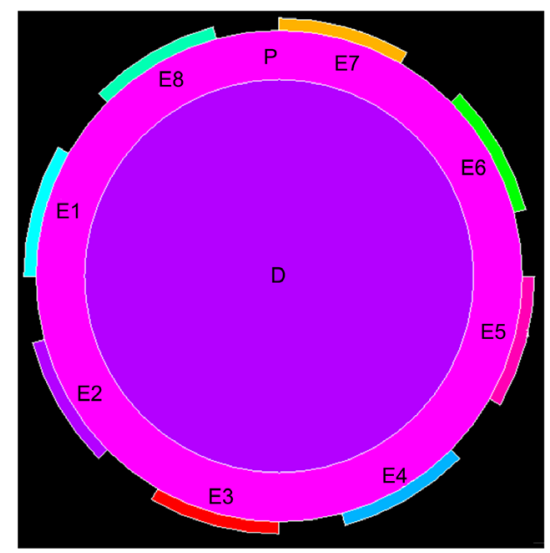

P -PVC PIPE

D -DIELECTRIC MEDIUM

E 1-E7 -ELECTRODES

Figure 2. Front view of designed sensor. 
the capacitance of all single electrode pairs is measured in the same way as before. Similarly, the remaining electrodes are energized one by one and the capacitance values are measured. Now, this will give a total number of 28 independent electrode combinations. In general, there are $N(N-1) / 2$ autonomous electrode combinations for an N-electrode sensor. There are, $M=n(n-1) / 2$ self-governing capacitance measurements for an $\mathrm{n}$ electrodes shown in Table 1 .

$$
\begin{gathered}
M=n(n-1) / 2, \text { When } n=8 \\
M=8(8-1) / 2 \\
M=8 \times 7 / 2 \\
M=28
\end{gathered}
$$

\subsection{Modelling Simulation Result}

In Figure 3, air medium is inside the pipe. Since, air has least dielectric constant conducting medium inside the pipe, considered as a low dielectric reference value. Because of its low conductivity the voltage distribution is not spread over the entire region inside the pipe.

In Figure 4 the pipe is completely filled with water and potential distribution is very wide comparing with air. Water is taken as high dielectric reference value in the algorithm using finite element method.

Table 1. Combination of sensor.

\begin{tabular}{cc}
\hline Electrode Excited & Capacitance Measured \\
\hline 1 & $\mathrm{C} 12, \mathrm{C} 13, \mathrm{C} 14, \mathrm{C} 15, \mathrm{C} 16, \mathrm{C} 17, \mathrm{C} 18$ \\
2 & $\mathrm{C} 23, \mathrm{C} 24, \mathrm{C} 25, \mathrm{C} 26, \mathrm{C} 27, \mathrm{C} 28$ \\
3 & $\mathrm{C} 34, \mathrm{C} 35, \mathrm{C} 36, \mathrm{C} 37, \mathrm{C} 38$ \\
4 & $\mathrm{C} 45, \mathrm{C} 46, \mathrm{C} 47, \mathrm{C} 48$ \\
5 & $\mathrm{C} 56, \mathrm{C} 57, \mathrm{C} 58$ \\
6 & $\mathrm{C} 67, \mathrm{C} 68$ \\
7 & $\mathrm{C} 78$ \\
\hline
\end{tabular}

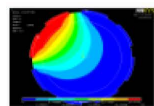

1

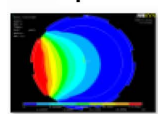

8

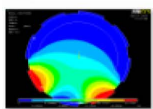

15

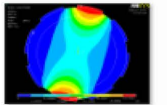

22

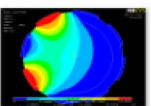

2

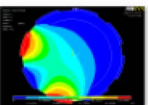

9

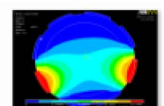

16

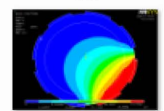

23

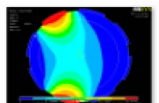

3

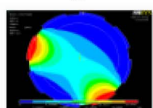

10

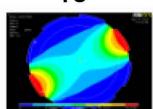

17

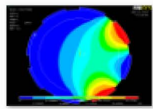

24

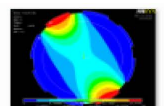

4

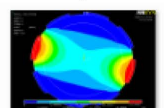

11

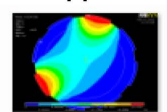

18

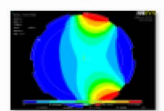

25

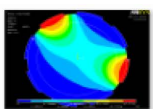

5

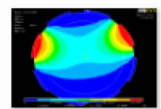

12

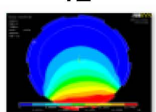

19

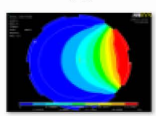

26

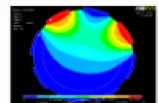

6

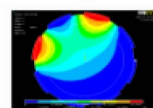

13

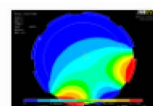

20

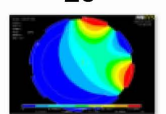

27

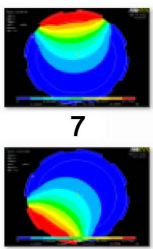

14

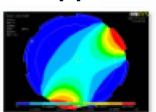

21

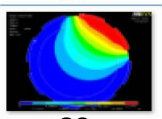

28

Figure 3. Capacitor charges between the plates in air medium. 


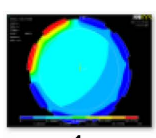

1

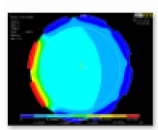

8

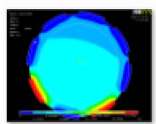

15

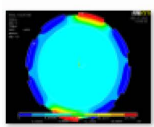

22

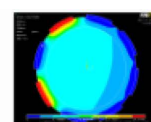

2

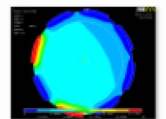

9

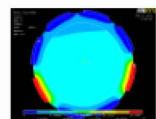

16

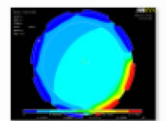

23

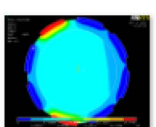

3

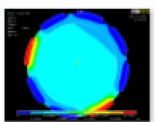

10

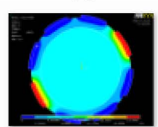

17

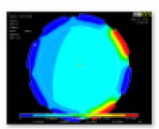

24

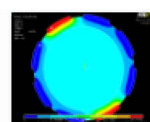

4

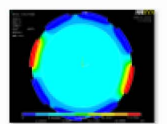

11

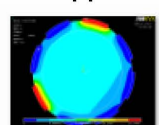

18

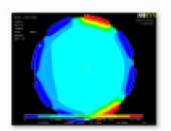

25

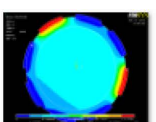

5

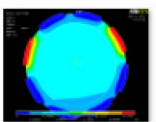

12

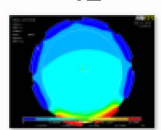

19

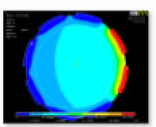

26

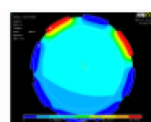

6

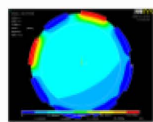

13

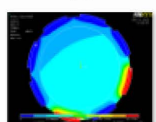

20

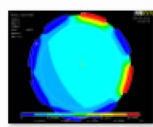

27

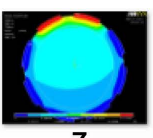

7

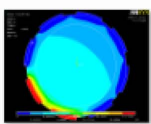

14

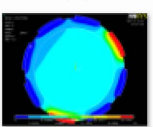

21

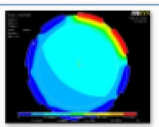

28

Figure 4. Capacitor charges between the plates in water medium.

\section{Implemented Real ECT System}

\subsection{Methodology}

The stable AC signal is used model to supply fixed frequency and constant voltage to each combinational electrode of the ECT system shown in Figure 5. The current flows start in this method, when applying to load from input voltage source. Obviously the charges accumulate on the electrodes and during negative half cycle time the electrodes immediately discharge not take so much time. The current flow changes by permittivity of the medium inside the electrodes. The reactance of the capacitor is minimized by using this technique.

If $X_{c}=$ capacitive reactance

$$
X_{c}=\frac{1}{2 \pi F C}
$$

Therefore, if $F=20 \mathrm{kHz}, X_{c}$ will have a minimum value. Thus it is found that, if $F$ value is increased, the $X_{c}$ value will be negligible. This indicates that increase in the switching speed of the combinational electrode ensures a high quality of the output signal. Using this signal, we can construct a clear image.

\subsection{Calibration Scheme}

The calibration scheme for ECT system could be achieved by the following procedure. The standard capacitor available in the market can be chosen for the calibration which is accomplished by the method of placing the fixed capacitor instead of the sensor and by measuring the corresponding voltage for the capacitance. Thus different equivalent can be measured for set of capacitor by keeping this equivalent voltage as a standard signal. A formula for calculating the capacitor based on the multiple polynomial regressive methods is derived.

$$
C=\left(K_{1} V_{n}\right)+\left(K_{2} V_{n-1}\right)+\cdots+\left(K_{n} V_{0}\right)
$$




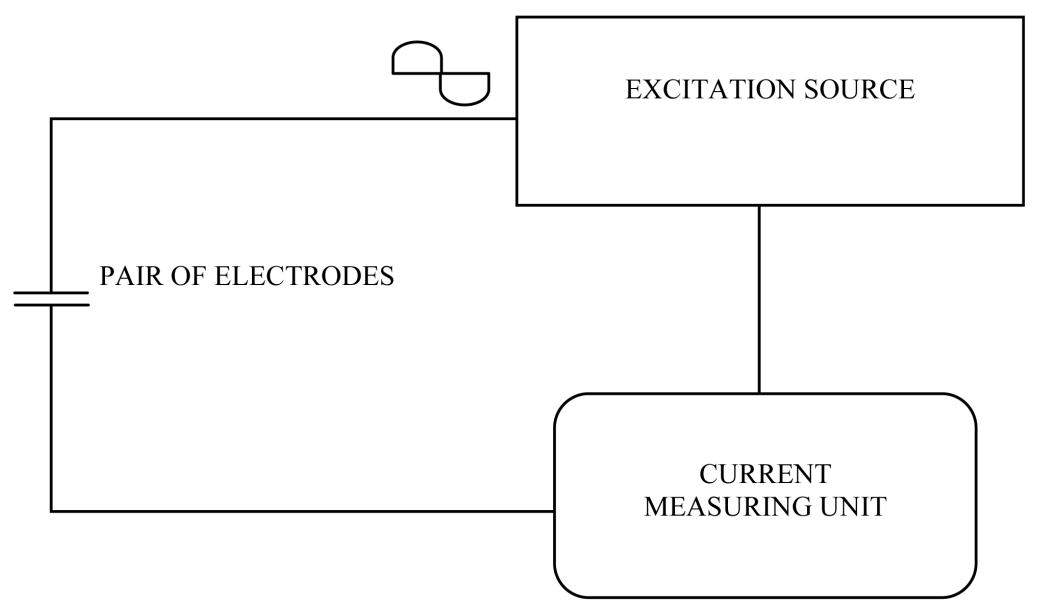

Figure 5. Block diagram of AC signal model.

Now, by replacing the designed sensor in place of fixed capacitor, it is possible to take the output voltages corresponding to the combinational electrode. By substituting this voltage in equation 3, the effective calibration of ECT sensor could be done. The proposed method of calibration clearly shows the elimination of other parameter calculation. Also it yields very accurate reading, and its offers simple operation.

\subsection{Signal Condition Circuit}

The output of the current sensing circuit is in the form of current which can be converted into a voltage using a converter circuit shown in the Figure 6 . The current is directly proportional to the voltage. The output of the circuit can be automatically displayed using the sensor. The current is converted into voltage using converter circuit. Then converter circuit is connected to a PC, and the result is displayed using Lab VIEW.

Current sensor detects the current and converts it into an easily measurable output voltage, which is proportional to the current through the measured lane. There are a broad range of sensors, and each sensor is suitable for a specific current range and environmental situation. None of the one sensor is best possible for all applications. Along with these sensors, a current sensing resistor is the most frequently used. It can be considered as a current-to-voltage converter. A resistor placed in the current path converts the current into voltage in a linear way. The current sensor connects the sensing resistor linking the load and the ground. Generally, op amp circuits are used to obtain an amplified output voltage, since the sensed voltage signal (VSEN $=$ ISEN $\times$ RSEN) is very small. In real time, the capacitive sensor is connected to the current to voltage converter, in which the sensed current from the capacitive sensor is converted into voltage. This voltage signal is interface to NI c DAQ 9174 using Lab VIEW.

\subsection{Formulation of Image Retrieval}

The ECT is use to calculate the potential distribution in capacitance by Laplace's equation [15], 


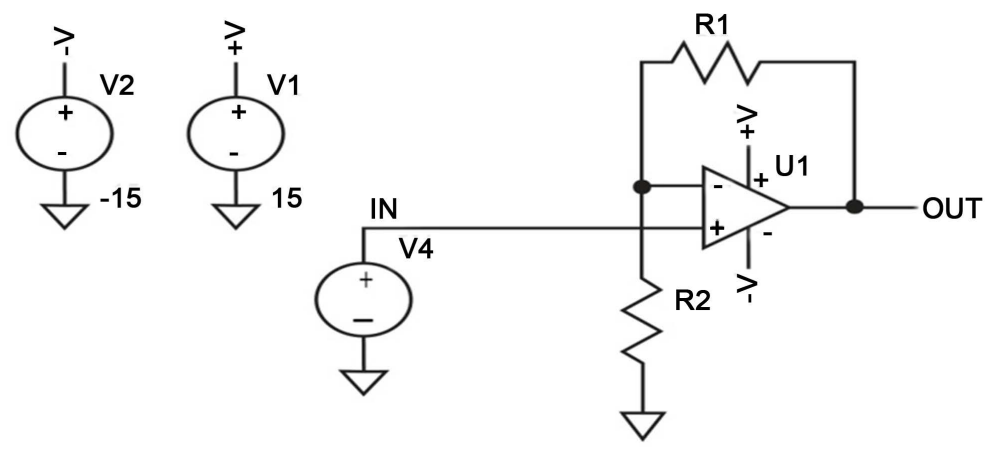

Figure 6. Current to voltage converter.

$$
\nabla\left[\varepsilon_{0} \varepsilon_{r}(y, z) \nabla \phi(y, z)\right]=0
$$

where,

$\varepsilon_{0}$ - The permittivity of free space,

$\varepsilon_{r}(y, z)$-The relative permittivity of the medium,

$\varphi(y, z)$ - The potential distribution in two dimensions.

The current flowing into the capacitor in proposed AC model ECT system is,

$$
\begin{gathered}
I_{c(t)}=\frac{\mathrm{d} q}{\mathrm{~d} t} \\
q=c v_{c}
\end{gathered}
$$

where, $v_{c}$-The potential variation between the source electrode and the detecting electrode.

According to the finite difference method used in calculation of potential distribution, the cross sectional retrieval image represented by the gray level $G(p)$. The gray level for the pixel $\mathrm{p}$ can be calculated from the following equation,

$$
G(p)=\frac{\sum_{k=0}^{7} \sum_{l=k+1}^{8} C_{k l}^{n} S_{k l}(P)}{\sum_{k=0}^{7} \sum_{l=k+1}^{8} S_{k l}(P)}
$$

$P=$ the sequence number of pixels,

$k=$ the sequence number of the source electrode,

$L=$ the sequence number of the detecting electrode,

$C_{k l}^{n}$ is the normalized capacitance,

$S_{k l}(P)$ is the relative capacitance change between electrode pair $k-1$.

\section{Experimental Analysis}

\subsection{Interface ECT Sensor with System}

The entire setup for the ECT system is connected as shown in Figure 7 based on the block diagram (Figure 1). The design could be achieved by using both software and a hardware model. The signal from the sensor is fed to the embedded controller AT MEGA series. The embedded controller switches the combinational electrodes, where 


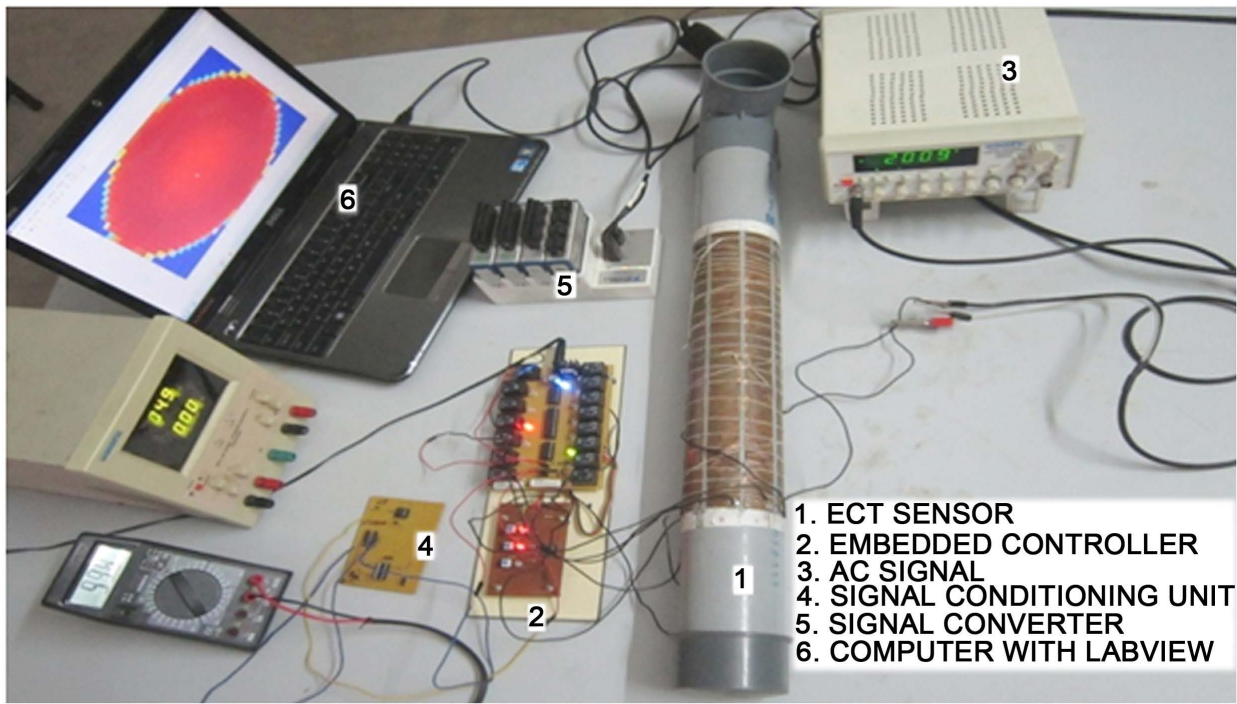

Figure 7. Implemented AC ECT system experimental setup.

the switching speed can effectively be increased in terms of micro seconds. The proposed AC ECT system with $20 \mathrm{kHz}$ method used to reduce the charging and discharging time. The pair of electrode measured current in serially, when the input source supply to ECT. Each combination of electrodes is furnished different current signal which can be obtained in higher speed by using micro controller. The output of the controller is then given to the signal conditioning unit, where scaling and multiplication of the received signal could be achieved with the help of a broadband operational amplifier IC. The current signal is converted into its equivalent voltage for further process simulation with LAB VIEW in real time. With LAB VIEW, the voltage for the real time sensor can be measured. From this, it can be clearly seen that the capacitive equivalence is matching the calibrated value. The DC voltage increase proportionally to the measured capacitance value. Thus the experimental result is confirmed. There is an increase in speed in the proposed ECT system. The water medium of output voltage values are shown in Table 2 and plotted in Figure 8.

\subsection{Embedded System}

An Embedded system is a computer system used for a particular function within a larger mechanical or electrical system, usually with real time computing limit. It is handle a particular task with dedication. The proposed ECT system increases the switching speed of the combinational capacitor as a set of relays in the input with the help of an embedded system is depicted in Figure 9. The relay circuit is driven by the higher current values of ULN2003A. It can be used to make a choice the source and detector electrode with high speed. The constant AC signal is sent to embedded controller, then it selects the electrode with the help of an Embedding program and Switches. The sensing current is interfaced with a Signal conditioning circuit which converts current to voltage with an input voltage of $15 \mathrm{~V}$ with $20 \mathrm{KHz}$ of AC embedded system. The standard current values are taken for the different dielectric media like water, air. Here water acts 
Table 2. Voltage (V) measurement of water for ECT sensor.

\begin{tabular}{cccccccc}
\hline DS & $\mathbf{2}$ & $\mathbf{3}$ & $\mathbf{4}$ & $\mathbf{5}$ & $\mathbf{6}$ & $\mathbf{7}$ & $\mathbf{8}$ \\
\hline 1 & 3.82 & 3.17 & 2.71 & 2.11 & 2.20 & 2.85 & 3.45 \\
2 & - & 3.72 & 3.11 & 2.69 & 2.56 & 3.04 & 3.30 \\
3 & - & - & 3.69 & 3.14 & 2.76 & 2.54 & 2.65 \\
4 & - & - & - & 3.68 & 3.09 & 2.65 & 2.55 \\
5 & - & - & - & - & 3.66 & 3.08 & 2.69 \\
6 & - & - & - & - & - & 3.64 & 3.06 \\
7 & - & - & - & - & - & - & 3.72 \\
\hline
\end{tabular}

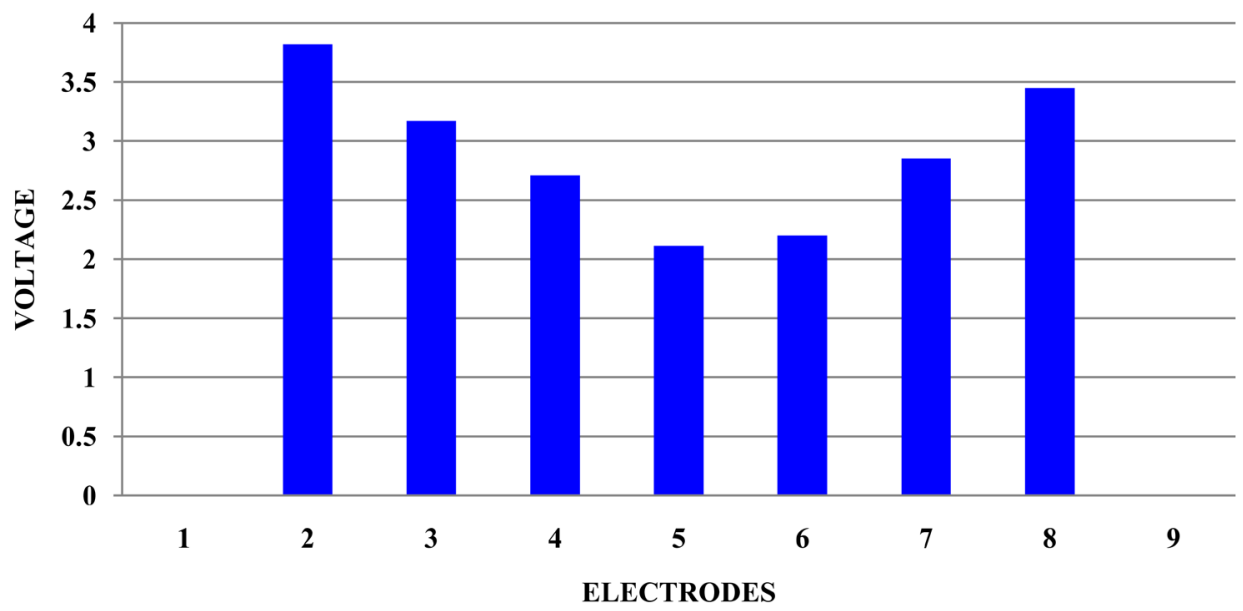

Figure 8. Electrode versus voltage for water medium.

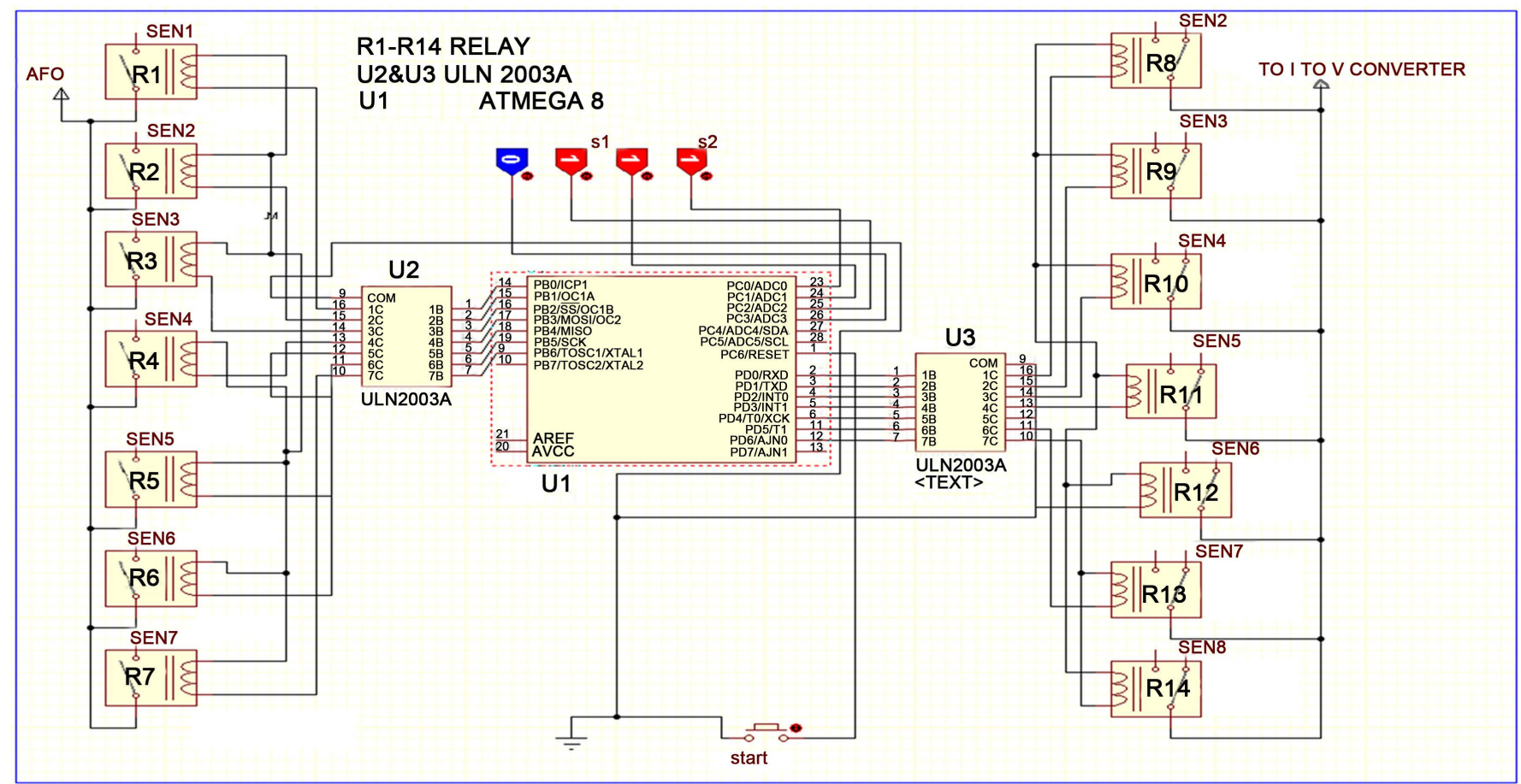

Figure 9. The circuit diagram of embedded system. 
as a dielectric medium in between the capacitive sensor. By indicating the voltage values, the capacitive sensor is connected to the signal condition circuits. The analog switches can be activated through Embedded programming to improve overall speed of the system.

\section{Image Reconstruction in Lab VIEW}

In real time implementation of ECT system, ECT sensor is connected to current to voltage converter, in which the sensed current from the capacitance sensor is converted into voltage through signal condition circuit. The voltage is connected to NI DAQ 9174 using Lab VIEW. The DAQ assistance gets the output in the form of dynamic array. Normally the DAQ receives signal in dynamic form, array is converted into cluster and output is displayed in image. The scaling is included for better result. The output of the system is shown below, which deficits the voltage variation corresponding to the selection of electrodes. In a scanning frame, depending on the total number of capacitance values formed by all electrodes, the switching control sub VI divides the process into-individual measurement steps. The digital I/O (DIO) ports control the connection of each electrode and the 8-1 multiplexer (MUX) in the measurement circuitry. The voltage signal that is processed by a pre-amp and lock-in amp is sampled by the data Sampling sub VI. After sampling all the capacitance values for a complete frame, these values are normalized in the Data Normalization and re-sorted into the form of matrix. By the Permittivity Calculation sub VI, these data are combined with the sensitivity matrix through the mesh generation, Image Generation, \& Image Display sub VI's, the data are finally converted in to an image that represents the material permittivity distribution. By looping the whole process frame by frame, the measurement circuit is controlled by the sub VI and it continuously samples the signal for displaying the dynamics of monitored process using Lab VIEW. The fully water and half water images are reconstructed through this block diagram Figure 10.

The Figure 11 shows an image of medium inside a circular pipe, execute using real time system. Dark portions represents Voltage potential is high due to the relative permittivity of the water is high and Light portions represents Voltage potential is low due to the relative permittivity of the air is low. These images are easy to identify and analyses the air and water medium inside the pipe with static or dynamic.

\section{Conclusion}

The ECT experimental study performed shows that the capacitance value in the range of picofarad can be measured and the system's corresponding voltage ranges from 1 volt to 4 volt. The output depicted is implementing real time system and the response is exactly similar to that of modeling using ANSYS. The switching speed of each capacitor is increased by eliminating the stray capacitance with the help of constant frequency. The AC based capacitance measurement reduces the problem of drift and achieves elevated signal to noise ratio. The circuit sensitivity of the capacitance between the detecting electrode and outer shield decreases with an increase in the speed of the signal 


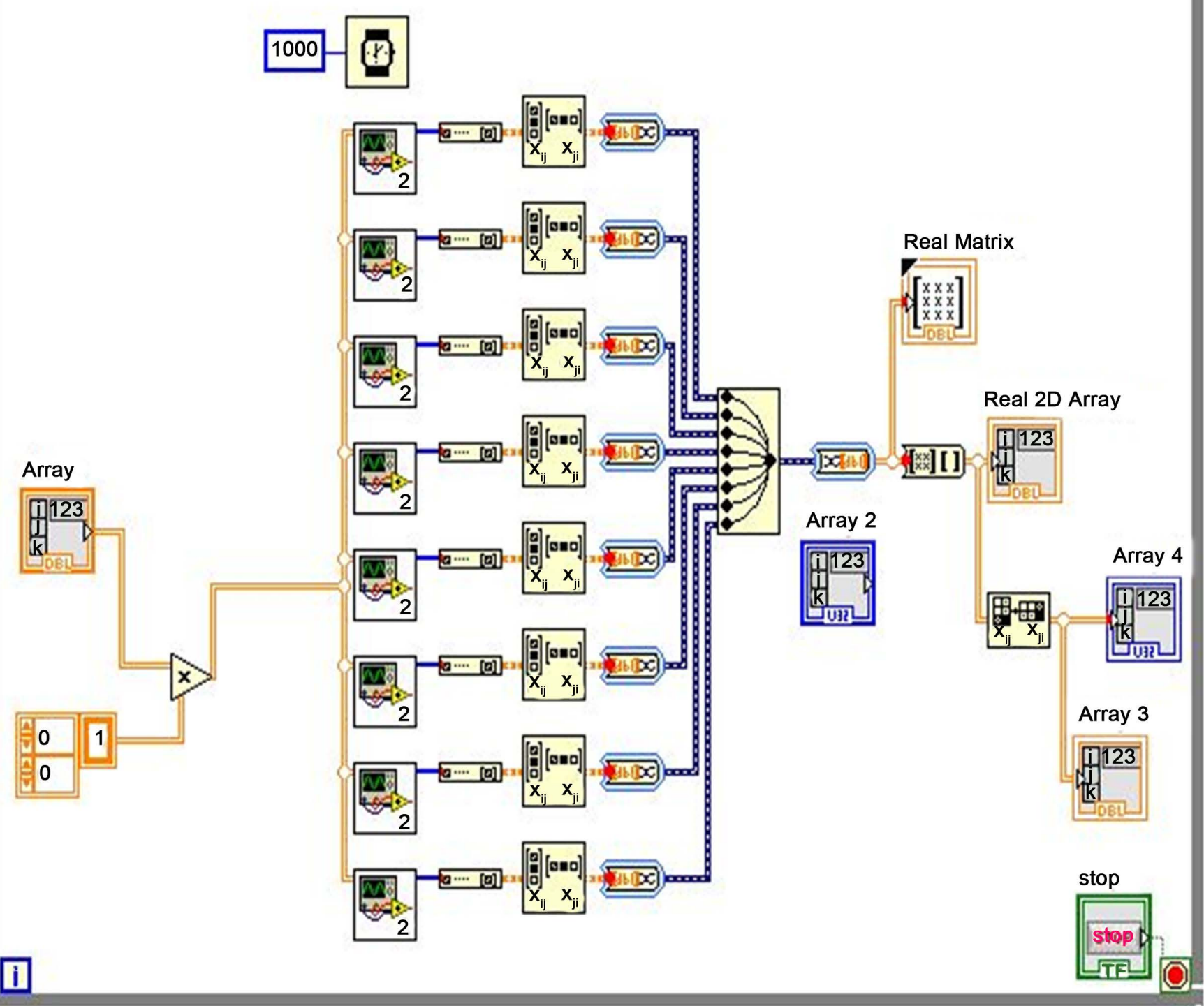

Figure 10. Lab VIEW block diagram.

AIR and WATER FULLY WATER

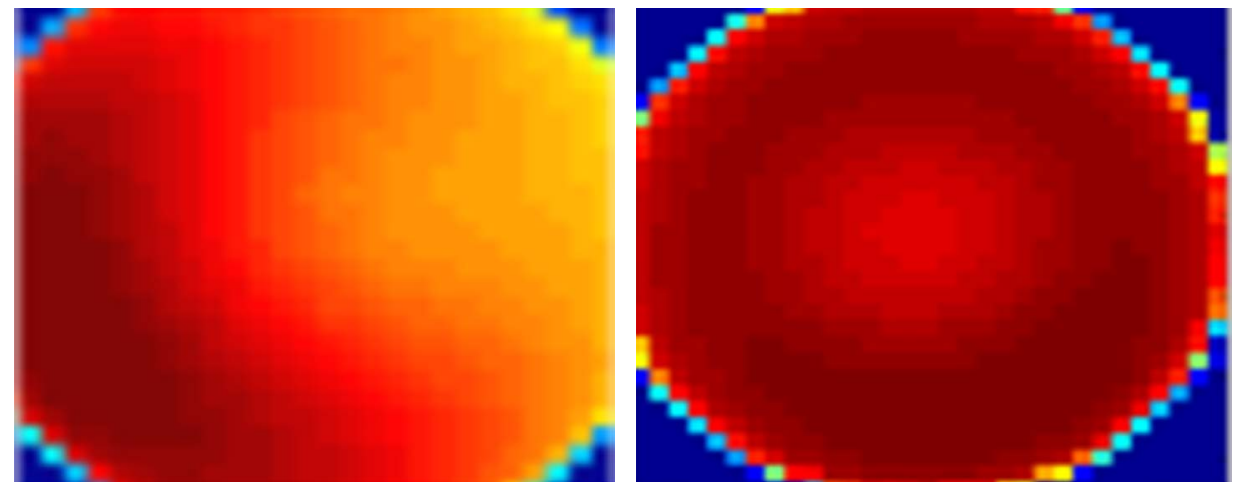

Figure 11. Image obtained in Lab VIEW as contain pipe. 
conditioning amplifier. Finally the image reconstructed of the permittivity medium inside the tube using Lab VIEW.

\section{References}

[1] Gao, Y.-L. and Zhang, Y.G. (2009) Key Issues in Designing High-Speed Hardware for Electrical Capacitance Tomography System. International Form on Computer Science Technology and Application, 25-27 December 2009, 332-335.

[2] Alme, K.J. and Mylvaganam, S. (2006) Electrical Capacitance Tomography-Sensor Models, Design, Simulations, and Experimental Verification. IEEE Sensor Journal, 6, 1256-1265. http://dx.doi.org/10.1109/JSEN.2006.881409

[3] Warsito, W., Marashdeh, Q. and Fan, L.-S. (2007) Electrical Capacitance Volume Tomography. IEEE Sensors Journal, 7, 525-535. http://dx.doi.org/10.1109/JSEN.2007.891952

[4] Wang, Q., Wang, H.X., Hao, K.H. and Dai, P. (2011) Two-Phase Flow Regime Identification Based on Cross-Entropy and Information Extension Methods for Computerized Tomography. IEEE Transactions on Instrumentation and Measurements, 60, 488-495. http://dx.doi.org/10.1109/TIM.2010.2058591

[5] Huang, S.M., Xie, C.G., Thorn, R., Snowden, D. and Beck, M.S. (1992) Design of Sensor Electronics for Electrical Capacitance Tomography. IEE Proceedings-G-Circuits, Device and System, 139, 83-88. http://dx.doi.org/10.1049/ip-g-2.1992.0014

[6] Sathyamoorthy, S. and Sarachandrababu, J. (2007) Design of High-Speed Pulse Input Based Capacitance Measurement for Electrical Capacitance Tomography. Sensors \& Transducers Journal, 75, 896-903.

[7] Fan, Z.Y. and Gao, R.X. (2010) A New Sensing Method for Electrical Capacitance Tomography. IEEE Transaction on Instrumentation and Measurement, 55, 415-428.

[8] Ji, Y. (2009) Design and Implementation of Hardware System for Electrical Capacitance Tomography. First International Workshop on Education Technology and Computer Science, Wuhan, 7-8 March 2009, 588-594. http://dx.doi.org/10.1109/etcs.2009.139

[9] Li, L.Y., Gao, M. and Chen, D.Y. (2011) A Novel Multiple Electrodes Excitation Method for Electrical Capacitance Tomography System. 2011 6th International Forum on Strategic Technology (IFOST), Harbin, 22-24 August 2011, 1167-1171.

[10] Fan, Z.Y. and Gao, R.X. (2012) A Frequency Selection Scheme for Increased Imaging Speed in ECT. 2012 IEEE International Conference on Imaging Systems and Techniques (IST), Manchester, 16-17 July 2012.

[11] Xu, L.J., Zhou, H.L., Cao, Z. and Yang, W.Q. (2013) A Digital Switching Demodulator for Electrical Capacitance Tomography. IEEE Transactions on Instrumentation and Measurements, 62, 1025-1033. http://dx.doi.org/10.1109/TIM.2012.2236731

[12] Teniou, S., Meribout, M. and Belarbi, K. (2012) Real-Time Reconstruction of Moving Objects in an Electrical Capacitance Tomography System Using Inter-Frame Correlation. IEEE Sensors Journal, 12, 2517-2525. http://dx.doi.org/10.1109/JSEN.2012.2194735

[13] Li, X., Huang, Z.Y., Wang, B.L. and Li, H.Q. (2009) A New Method for the Online Voidage Measurement of the Gas-Oil Two-Phase Flow. IEEE Transaction on Instrumentation and Measurement, 58, 1571-1577. http://dx.doi.org/10.1109/TIM.2008.2009138

[14] Yang, W.Q., Stott, A.L. and Gamio, J.C. (2003) Analysis of the Effect of Stray Capacitance on an AC-Based Capacitance Tomography Transducer. IEEE Transaction on Instrumentation and Measurement, 52, 7-12. http://dx.doi.org/10.1109/tim.2003.817925

[15] Moura, L.F.M., Cenedese, E. and Azevedo Filho, A.C. (2007) Numerical Study of a Capacitive Tomography System for Multiphase Flow. Thermal Engineering, 8, 67-78. 
Submit or recommend next manuscript to SCIRP and we will provide best service for you:

Accepting pre-submission inquiries through Email, Facebook, LinkedIn, Twitter, etc. A wide selection of journals (inclusive of 9 subjects, more than 200 journals)

Providing 24-hour high-quality service

User-friendly online submission system

Fair and swift peer-review system

Efficient typesetting and proofreading procedure

Display of the result of downloads and visits, as well as the number of cited articles

Maximum dissemination of your research work

Submit your manuscript at: http://papersubmission.scirp.org/

Or contact cs@scirp.org 